\title{
Gender Effect on the Relation between Diabetes and Hospitalization for Heart Failure
}

Authors

Affiliations

\section{Seghieri ${ }^{1}$, P. Francesconi ${ }^{2}$, S. Cipriani ${ }^{2}$, M. Rapanà ${ }^{3}$, R. Anichini ${ }^{4}$, F. Franconi ${ }^{5}$, S. Del Prato ${ }^{6}$, G. Seghieri ${ }^{4}$}

Affiliation addresses are listed at the end of the article
Key words

- heart failure

- hospitalization

- diabetes mellitus

gender received 17.04.2011 first decision 30.06.2011 accepted 13.07.2011

\section{Bibliography}

Dol http://dx.doi.org/

10.1055/s-0031-1284377

Published online:

September 13, 2011

Exp Clin Endocrinol Diabetes

2012; 120: 51-55

J. A. Barth Verlag in

(c) Georg Thieme Verlag KG

Stuttgart · New York

ISSN 0947-7349

\section{Correspondence}

\section{G. Seghieri, MD}

Department of Internal

Medicine,

Spedali Riuniti,

Viale Matteotti 9/D

51100 Pistoia

Italy

Tel.: +39/33/8694 1642

Fax: $+39 / 057 / 3352005$

$+39 / 057 / 33533150$

gseghier@tin.it

\section{Abstract}

$\nabla$

Aims: Cardiovascular risk among diabetic patients is at least twice as much the one for nondiabetic individuals and even greater when diabetic women are considered. Heart failure (HF) is a common unfavorable outcome of cardiovascular disease in diabetes. However, since the comparison among sexes of heart failure prevalence in diabetic patients remains limited, this study is aimed at expanding the information about this point.

Methods: We have evaluated the association between diabetes and HF by reviewing the medical records of all subjects discharged from the Internal Medicine and Cardiology Units of all hospitals in the Tuscany region, Italy, during the period January 2002 through December 2008. In

\begin{tabular}{ll}
\hline \multicolumn{2}{l}{ Abbreviations } \\
$\nabla$ \\
$\mathrm{CI}$ & confidence interval \\
$\mathrm{RR}$ & relative risk \\
$\mathrm{HF}$ & heart failure \\
ICD-9 & International Classification of Diseases- \\
& Ninth Revision-Clinical Modification
\end{tabular}

\section{Introduction}

$\nabla$

Heart failure (HF) is a major cause for hospitalization especially in the elderly, (American Heart Association Statistics Committee, 2009) and, at the same time, is strongly related to diabetes mellitus, a prominent cause of ischemic heart disease (Nichols et al., 2004; Nichols et al., 2001). Studies carried out in Iceland showed that the age-adjusted odds ratio for development of $\mathrm{HF}$ was $2.8(2.2-3.6)$ in diabetic patients as compared to non-diabetic individuals (Thrainsdottir et al., 2005). A difference in HF risk has been particular we sought concomitance of ICD-9-CM codes for diabetes and HF.

Results: Patients discharged by Internal Medicine were on average older, more represented by women, and had a lesser number of individuals coded as diabetic ( $\mathrm{p}<0.05$ for all). Relative risk for HF (95\% CI) was significantly higher in patients with diabetes, irrespective of gender 1.39 (1.36$1.41)$ in males; 1.40 (1.37-1.42) in females. When the diabetes-HF association was analyzed according to decades of age, a "horse-shoe" pattern was apparent with an increased risk in 40-59 years old in female patients discharged by Internal Medicine.

Conclusions: Although there is not a difference in the overall HF risk between hospitalized male and female diabetic patients, women have an excess risk at perimenopausal age.

reported between men and women. In the Framingham study, HF risk was two-fold higher in men and five-fold higher in women with diabetes as compared to the non-diabetic population (Kannel et al., 1974). This effect was more apparent in younger subjects, e.g., under 65 years, where the risk of developing HF was four- and eight-fold higher in men and women with diabetes, respectively. In the NHANES and the Cardiovascular Health Study, diabetes was an independent risk factor for HF hazard ratios 1.85 (1.51-2.28) and 1.74 (1.38-2.19), respectively (He et al., 2001; Gottdiener et al. 2000). However, most of the available information on the epidemiology of HF and diabetes has been gathered in the American population. Given the difference in the cardiovsacular risk profile existing among different populations and geographical areas (Eichler et al., 2007), there is the need for local epidemiologic data, especially with regard to any sex difference. We have, therefore, analyzed all hospital discharges during a 7-year period in Tus- 
cany, a region of central Italy, by crossing ICD-9-CM codes for diabetes mellitus and heart failure to assess concomitance of these 2 conditions, searching for any eventual difference between genders.

\section{Materials and Methods}

\section{$\nabla$}

We have screened a regional centralized database recording all discharges from the Cardiology and Internal Medicine wards of the hospitals in Tuscany during the period 2002 through 2008. The database provided the whole region data as well as those from each of the 15 local health authorities. Based on the 2008 census, the total population of the Tuscany region was 3686377 inhabitants, all of them covered by the regional public health care program including a network of hospitals accounting for $>90 \%$ of the whole number of hospital admissions. The database was searched for concomitance in the discharge of the International Classification of Diseases, Ninth Revision (ICD9-CM) codes 250.xx (i.e., diabetes mellitus) in main or secondary diagnosis and ICD-9-CM codes 401.91, 402.01, 402.11, 402.91, 404.01, 404.3, 404.13, 404.93, 428.0, 428.1, 428.9 (i.e., heart failure) in both main and secondary diagnosis as well (Nichols et al., 2001). Analysis was limited to discharges of alive patients from Cardiology and Internal Medicine Units because the vast majority of patients with heart failure are admitted to these wards. The proportion of diagnosis of diabetes was slightly, though statistically significant higher in the patients admitted to the Cardiology Units ( 15.1 vs. $14.6 \%, X^{2}=20.32 ; p=0.0001$; 0 Table 1). We have analyzed data only for patients with $\geq 30$ years of age because HF prevalence was very low in young subjects.

The study population was stratified for age group and sex and the Mantel-Haenszel Logit method was used to calculate the relative risk (RR) and 95\% Confidence Intervals for the diagnosis of diabetes in patients discharged with the diagnosis of HF. The sensitivity for the diagnosis of diabetes at discharge was calculated in 28198 patients by crossing the diabetes diagnosis in the discharge records and the one made via an independent source. In short: the records were crossed with those of a database containing sure diabetes diagnosis obtained by multiple sources (use of antidiabetic drugs, previous hospitalizations, legal certifications etc.). By this way sensitivity was $60.5 \%$ and $70.2 \%$ for patients discharged by Internal Medicine and Cardiology, respectively, with an Odds Ratio=1.29 $(1.19-1.40)$ for being diagnosed diabetic when present, for Cardiology. All analyses were performed with SAS software, version 8.2 for Windows (SAS Institute, Cary, NC).

The study was approved by the Ethical Committee of the Spedali Riuniti, Pistoia, Italy.

Table 1 Main characteristics of alive patients discharged by Internal Medicine or Cardiology wards of Tuscan Hospitals (years 2002-08).

\begin{tabular}{|llll|}
\hline & $\begin{array}{l}\text { Internal } \\
\text { Medicine }\end{array}$ & Cardiology & p \\
\hline age $(\mathrm{yr})( \pm \mathrm{SD})$ & $72.1 \pm 14.5$ & $68.5 \pm 12.1$ & 0.001 \\
\hline $\begin{array}{l}\text { no. }(\%) \text { of males } \\
\begin{array}{l}\text { no. }(\%) \text { of diabetes } \\
\text { discharges }\end{array}\end{array}$ & $361552(48.2)$ & $111818(65.2)$ & 0.0001 \\
\hline \begin{tabular}{l} 
no $(\%)$ of HF discharges \\
\hline
\end{tabular} & $110630(14.6)$ & $25865(15.1)$ & 0.0001 \\
\hline
\end{tabular}

\section{Results}

$\nabla$

The database consisted of 922164 records. The contribution to the whole study population was greater for the Internal Medicine $(81.4 \%)$ than for the Cardiology Units (18.6\%). The 2 populations showed some difference in gender distribution, age, and diabetes between discharges from the 2 units with a greater prevalence of male, older, and diabetic individuals in the Internal Medicine population ( $\bullet$ Table 1). On the contrary, the prevalence of HF was higher among patients discharged from the Cardiology Units ( Table 1).

Both in the Cardiology or in Internal Medicine discharges, the rate of HF diagnosis increased in a linear manner from the age of 30 to that of 90 years in both sexes ( 0 Table 2,3 ). On the contrary, the distribution of the diabetes diagnosis had a "horse shoe" shape with a nadir in the 60-79 year decades ( $\bullet$ Fig. 1 ). The overall RR for HF in patients with a diabetes diagnosis was about 1.40 with no difference between males and females ( Table 4). The overall risk, however, was slightly higher in the discharges from the Cardiology wards ( $\bullet$ Table 2, 3). Although the overall RR for HF was similar in man and women, a difference between genders became apparent when the diabetes/HF relationship was assessed based on age ( $\bullet$ Table 4 ) with an early rise of RR in women 40-59 years of age. This difference was entirely accounted for by the age-related RR observed in the discharges from the Internal Medicine units ( $\bullet$ Table 2, 3).

\section{Discussion \\ $\nabla$}

Assessment of the hospital discharges allows the evaluation of the association between diabetes and heart failure in a very large sample including more than 900000 observations over a 7 -year period. Although detailed information may be lacking, overall phenomena can be clearly identified. Thus, when discharges from Internal Medicine and Cardiology Units have been taken into account it was readily apparent that the frequency of diabetes and HF diagnosis had a different association with age. The prevalence rate of HF diagnoses increased in a linear manner from the age of 30 till the age of 90 until, while that of diabetes peaked in the $7^{\text {th }}$ decades of age followed by progressive decline. This difference is likely to be accounted for by late reduction in survival among diabetic individuals leading to a progressive enrichment of HF. These figures may be affected by the low sensitivity rate of diabetes diagnosis due to under-coding at discharge (Carral et al., 2003; Ragnarson-Tennvall et al., 2000) as well as to insufficient recognition of new cases of diabetes during the hospital stay (Levetan et al., 1998). However, when determined in more than 28000 cases, we found $60-70 \%$ sensitivity of the diagnosis of diabetes in hospital discharge. Prevalence of diabetes was, even if slightly, higher in patients discharged by Cardiology, part due to higher sensitivity for correct diagnosis, and probably part due to a frequent rate of more serious states frequently associated with hyperglicemia. Our database, however was not able to validate this latter hypothesis. Neither was our database able to differentiate between newly diagnosed diabetes and pre-admission diabetic diagnosis.

By crossing the 2 diagnosis, the RR of HF increased by $40 \%$ in the presence of the diagnosis of diabetes. This finding is in full agreement with previous epidemiological observations (Nichols et al., 2004). Similar to what previously observed, the association between the diagnosis of HF and that of diabetes had a 


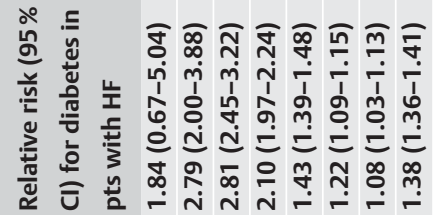

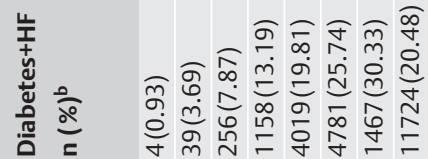

$\frac{y}{\frac{\tilde{\pi}}{\tilde{E}}}$

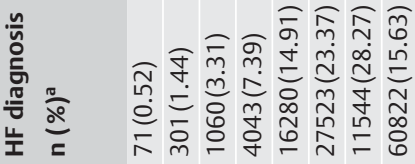

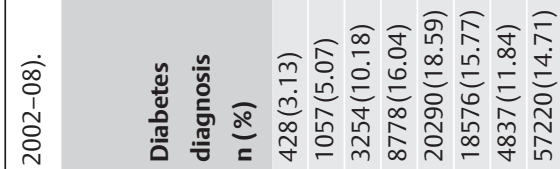

$\stackrel{n}{\stackrel{0}{\pi}}$

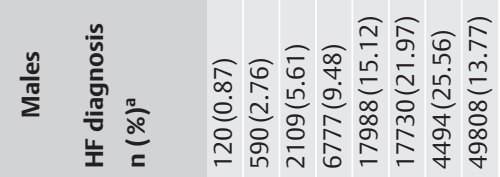

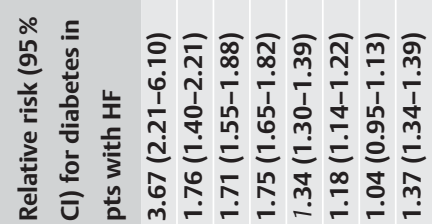

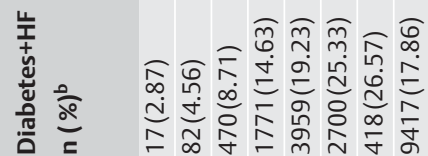

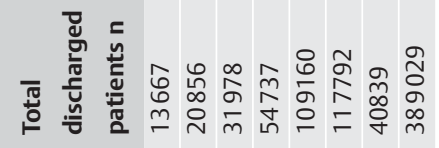

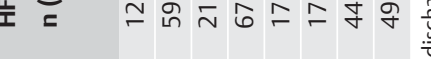

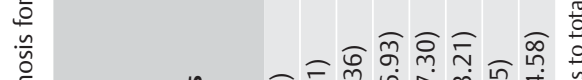

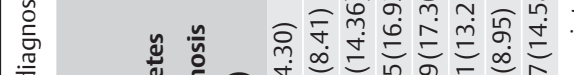

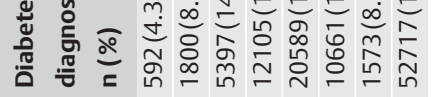

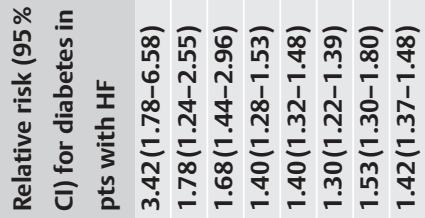

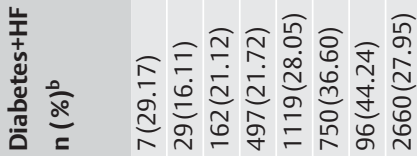

$\frac{y}{\tilde{\pi}}$

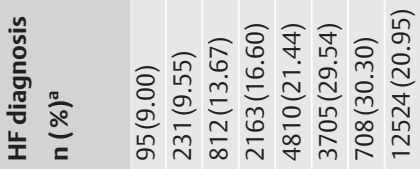

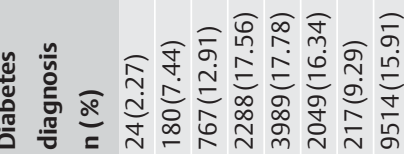
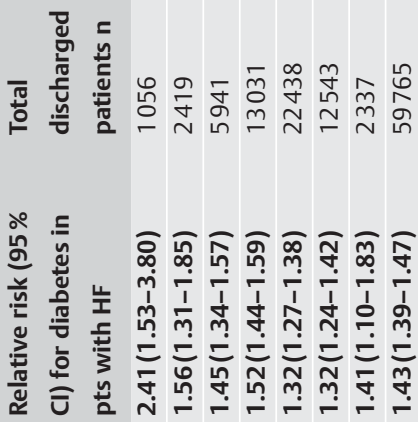

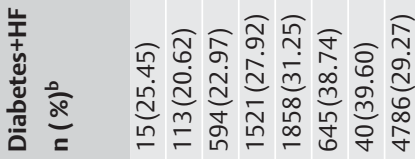

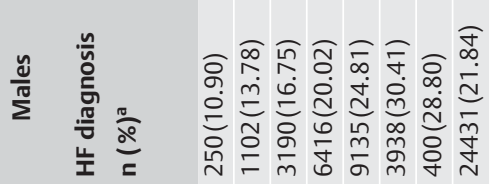

क人ิธ

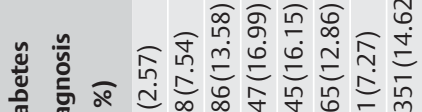
는 密 垔

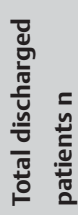




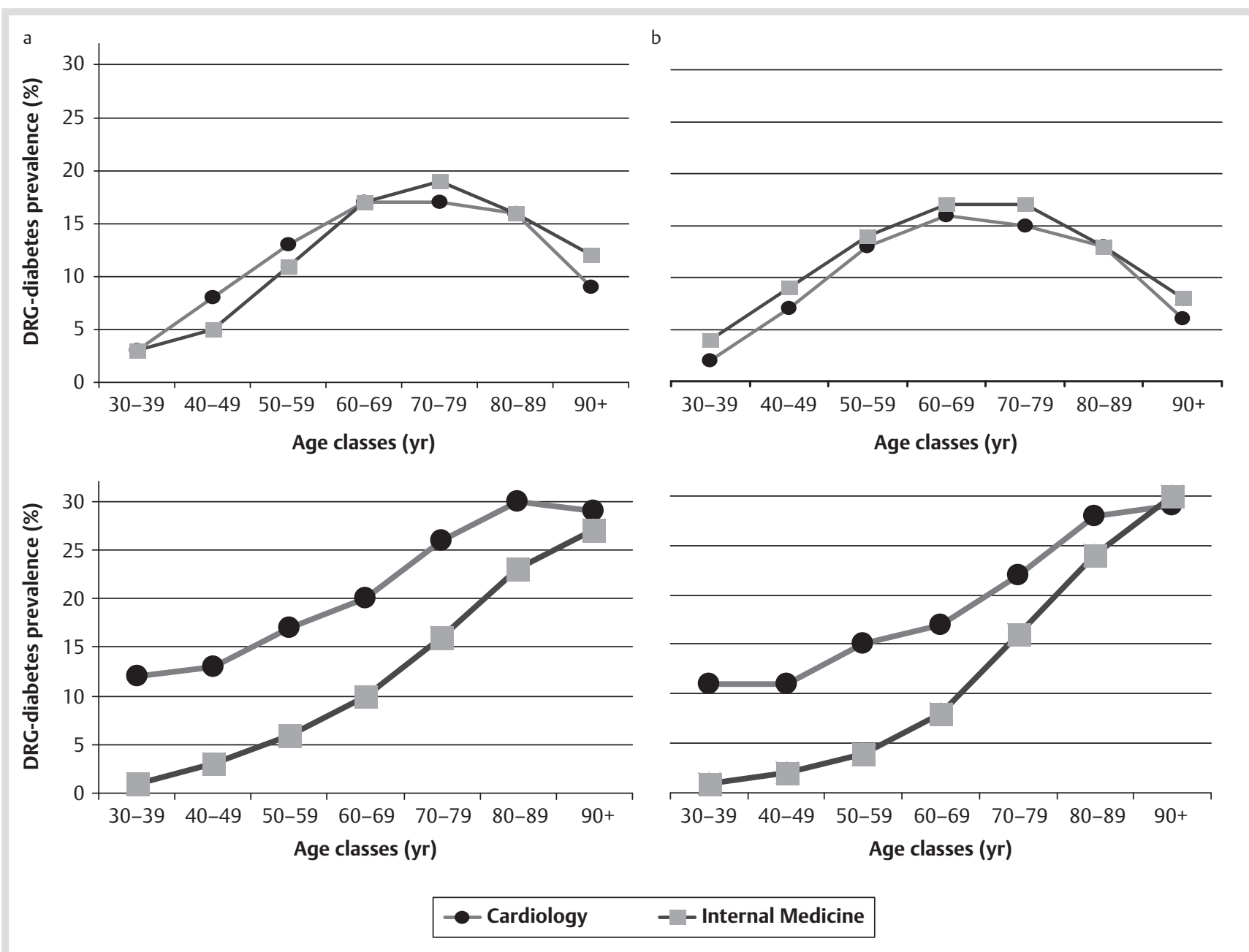

Fig. 1 Distribution of diabetes (top) and HF (bottom) hospitalization in female (a) and male (b) patients discharged by Cardiology and Internal Medicine in years 2002-08 across age groups.

Table 4 Relative risks expressed as $\mathrm{OR}(95 \% \mathrm{Cl})$ of being hospitalized for $\mathrm{HF}$ if affected from diabetes in alive discharged patients of both Internal Medicine and Cardiology, (years 2002-08) stratified for gender and age groups.

\begin{tabular}{|lll|} 
& Males & Females \\
\hline age-groups $(\mathbf{y r})$ & OR $(\mathbf{9 5} \% \mathrm{Cl})$ & \\
\hline $\mathbf{3 0 - 3 9}$ & $2.24(1.57-3.19)$ & $2.24(1.22-4.10)$ \\
\hline $\mathbf{4 0 - 4 9}$ & $1.56(1.36-1.80)$ & $2.61(2.03-3.45)$ \\
\hline $\mathbf{5 0 - 5 9}$ & $1.53(1.44-1.63)$ & $2.43(2.18-2.69)$ \\
$\mathbf{6 0 - 6 9}$ & $1.63(1.57-1.69)$ & $1.86(1.77-1.96)$ \\
\hline $\mathbf{7 0 - 7 9}$ & $1.33(1.29-1.36)$ & $1.42(1.38-1.46)$ \\
$\mathbf{8 0 - 8 9}$ & $1.20(1.17-1.24)$ & $1.14(1.12-1.17)$ \\
\hline $\mathbf{9 0}$ & $0.98(0.94-1.01)$ & $1.10(1.05-1.15)$ \\
\hline Total & $\mathbf{1 . 3 9}(\mathbf{1 . 3 6 - 1 . 4 1 )}$ & $\mathbf{1 . 4 0}(\mathbf{1 . 3 7 - 1 . 4 2})$ \\
\hline
\end{tabular}

characteristic "horse shoe" shape over a 70-year span age with a relatively early rise followed by a progressive decrease with advancing age (Nichols et al., 2004). This association was greater among women in the $4^{\text {th }}$ and $5^{\text {th }}$ decade of age as compared to the male gender. Our finding is much in line with the results of the Framingham Study (Kannel et al., 1976), as well as those of more recent surveys showing an increased impact of diabetes on the incidence of coronary heart disease (CHD) in women in the postmenopausal age (Barrett-Connor and Wingard, 1983; Barrett-Connor et al., 2004). Since CHD is the primary cause of HF in western population it is not surprising that HF prevalence follows the same pattern (Nichols et al., 2004; Nichols et al., 2001). Therefore, our findings are consistent with the hypothesis of a 'peri-menopausal effect', underscoring the development of a specific age-related vulnerability in women.

The difference in the association between diabetes and $\mathrm{HF}$ between gender in the $4^{\text {th }}$ and $5^{\text {th }}$ decade of age was particularly striking in the discharges from the Internal Medicine Units, while the phenomenon was not apparent when discharges from the Cardiology wards were considered. The main reason why this gender difference was not present in patients discharged by Cardiology could be ascribed to the diluting effect exerted by the much lower females/males ratio in patients admitted to Cardiology as compared to Internal Medicine, especially in younger age classes, in agreement with what had previously been observed (Houde et al., 2007). A further reason may be related to a certain degree of disparity between the 2 genders in the treatment of cardiovascular events. Many studies have, indeed, looked at differences between men and women with acute coronary syndrome (Juutilainen et al., 2004). These studies have moreover shown that women have worse outcomes, receive fewer invasive interventions, are more difficultly admitted to specialised clinics such as Cardiological settings (Houde et al., 2007) and experience delay in the initiation of established medical therapies. A further aspect is represented by the greater facility in confusing 
HF symptoms in women (Caruana et al., 2000; Cowie et al., 1999) leading to a potential reduced access of women to specialised hospital settings. The overall specular consequence of all these potential treatment disparities is expected to be on one hand the lack of any 'gender effect' in the association between diabetes and HF hospitalization in the more specialised Cardiology settings and on the other the observation that patients discharged from the Internal Medicine wards Units were more heterogeneous with a prevalence of females, older individuals and with a higher absolute number of diabetic patients.

Potential limitations and drawbacks of our analysis should be kept in mind. Besides the potential bias introduced by incomplete and/or inaccurate diagnosis of diabetes in the medical records, our database does not allow the identification of predicting factors for HF in diabetic individuals. Therefore, we cannot speculate on the cause(s) of the increased association between $\mathrm{HF}$ and diabetes and the reason(s) for the specific increase among younger women although coronary heart disease may be a very likely underlying predisposing condition (Nichols and Brown, 2002). Besides all these limitations, we believe that the size of the sample is large enough to support an 'age-related gender difference' in the association between diabetes and HF, suggesting that diagnostic and therapeutic strategies should be sought to address in more proper manner HF in males and females, also in the light of a larger burden of costs among hospitalized diabetic patients (Olveira-Fuster et al., 2004; Greenberg et al., 2010).

\section{Acknowledgements}

\section{$\nabla$}

This paper in abstract form has been partially presented as poster at the $46^{\text {th }}$ Annual Meeting, Stockholm, Sweden, 20-24 September 2010 and published as abstract in Diabetologia 2010:53 Suppl1S170.

This work was supported by a grant of the Fondazione Cassa di Risparmio di Pistoia e Pescia, Pistoia, Italy.

Declaration of Competing Interests: The authors declare that they have no conflict of interest.

\footnotetext{
Affiliations

${ }^{1}$ Scuola Superiore S. Anna, MES Laboratory, University of Pisa, Pisa, Italy

${ }^{2}$ Agenzia Regionale Sanità, Regione Toscana, Florence, Italy

${ }^{3}$ Epidemiology Unit, Spedali Riuniti, Pistoia, Italy

${ }^{4}$ Department of Internal Medicine, Spedali Riuniti, Pistoia, Italy

${ }^{5}$ Department of Pharmacology, University of Sassari, Sassari, Italy

${ }^{6}$ Department of Endocrinology and Metabolism, Section of Metabolic

Diseases and Diabetes, University of Pisa, Pisa, Italy
}

\section{References}

1 American Heart Association Statistics Committee and Stroke Statistics Subcommittee. Heart disease and stroke statistics - 2009 update: a report from the American Heart Association Statistics Committee and Stroke Statistics Subcommittee. Circulation 2009; 119: e21-e181

2 Barrett-Connor E, Giardina EG, Gitt AK et al. Women and heart disease: the role of diabetes and hyperglycemia. Arch Intern Med 2004; 164: 934-942
3 Barrett-Connor E, Wingard DL. Sex differential in ischemic heart disease mortality in diabetics: a prospective population based study. Am J Epidemiol 1983; 118: 489-496

4 Carral F, Olveira G, Aguilar M et al. Hospital discharge records underreport the prevalence of diabetes in inpatients. Diabetes Res Clin Pract 2003; 59: 145-151

5 Caruana L, Petrie MC, Davie AP et al. Do patients with suspected heart failure and preserved left ventricular systolic function suffer from "diastolic heart failure" or from misdiagnosis? A prospective descriptive study. BMJ 2000; 321: 215-218

6 Cowie MR, Wood DA, Coats AJ et al. Incidence and aetiology of heart failure; a population-based study. Eur Heart J 1999; 20: 421-428

7 Eichler K, Puhan MA, Steurer J et al. Prediction of first coronary events with the Framingham score: a systematic review. Am Heart J 2007; 153: 722-731

8 Gottdiener JS, Arnold AM, Aurigemma GP et al. Predictors of congestive heart failure in the elderly: the Cardiovascular Health Study. J Am Coll Cardiol 2000; 35: 1628-1637

9 Greenberg MR, Bond WF, Mackenzie RS et al. Gender disparity in emergency department Non-ST Elevation Myocardial Infarction. J Emerg Med 2010; Sep 29 [Epub ahead of print]

$10 \mathrm{He} J$, Ogden LG, Bazzano LA et al. Risk factors for congestive heart failure in US men and women: NHANES-I epidemiologic follow-up study. Arch Intern Med 2001; 161: 996-1002

11 Houde S, Ehrmann Feldman D, Pilote L et al. Are there sexrelated differences in specialized, multidisciplinary congestive heart failure clinics? Can J Cardiol 2007; 23: 451-455

12 Juutilainen $A$, Kortelainen $S$, Lehto $S$ et al. Gender difference in the impact of type 2 diabetes on coronary heart disease risk. Diabetes Care 2004; 27: 2898-2904

13 Kannel WB, Hjortland M, Castelli WP. Role of diabetes in congestive heart failure: The Framingham study. Am J Cardiol 1974; 34: 29-34

14 Kannel WB, Hjortland MC, McNamara PM et al. Menopause and risk of cardiovascular disease: the Framingham study. Ann Intern Med 1976; 85: 447-452

15 Levetan CS, Passaro M, Jablonski K et al. Unrecognized diabetes among hospitalized patients. Diabetes Care 1998; 21: 246-249

16 Nichols GA, Brown JB. The impact of cardiovascular disease on medical care costs in subjects with and without type 2 diabetes. Diabetes Care 2002; 25: 482-486

17 Nichols GA, Gullion CM, Koro CE et al. The incidence of congestive heart failure in type 2 diabetes: an update. Diabetes Care 2004; 27: 18791884

18 Nichols GA, Hillier TA, Erbey JR et al. Congestive heart failure in type 2 diabetes: prevalence, incidence, and risk factors. Diabetes Care 2001; 24: 1614-1619

19 Olveira-Fuster G, Olvera-Márquez P, Carral-Sanlaureano F et al. Excess hospitalizations, hospital days, and inpatient costs among people with diabetes. Diabetes Care 2004; 27: 1904-1909

20 Ragnarson-Tennvall G, Apelqvist J, Eneroth M. The inpatient care of patients with diabetes mellitus and foot ulcers. A validation study of the correspondence between medical records and the Swedish Inpatient Registry with the consequences for cost estimations. J Intern Med 2000; 248: 397-405

21 Thrainsdottir IS, Aspelund T, Thorgeirsson G et al. The association between glucose abnormalities and heart failure in the populationbased Reykjavik study. Diabetes Care 2005; 28: 612-616 